\title{
Thermal stress analysis of functionally graded material structures using analytical expressions in radial integration $\mathrm{BEM}$
}

\author{
K. Yang, Z. C. Yuan \& J. Lv \\ State Key Laboratory of Structural Analysis for Industrial Equipment, \\ Dalian University of Technology, China
}

\begin{abstract}
This paper presents a new approach using analytical expressions in the radial integration boundary element method (RIBEM) for 2D thermal stress analysis of structures consisting of functionally graded materials. This approach can improve the computational efficiency considerably and can overcome the timeconsuming deficiency of RIBEM in computing involved radial integrals. Using this equation together with the three-step multi-domain BEM technique, thermal stress analysis can be carried out for complicated structures comprised of, arbitrarily, many numbers of media with varying material properties. The Kelvin fundamental solutions independent of elastic modulus are used in this paper. As a result, the resulting integral equations include domain integrals due to the nonhomogeneity and temperature variations of the material. All these domain integrals are transformed into equivalent boundary integrals using the radial integration method (RIM), resulting in a pure boundary element analysis algorithm. Numerical examples are given to demonstrate the efficiency of the presented approach.
\end{abstract}

Keywords: functionally graded material, thermal stress analysis, boundary element method, radial integration method.

\section{Introduction}

Functionally graded materials (FGMs) are composite materials made of two or more material constituents (usually metal/ceramic constituents). In these materials, fractions of phases vary continuously in space and, therefore, macroscopic thermal and mechanical properties of FGMs exhibit a graded 
behavior of continuous change [1]. Due to acute changes in temperature, thermal stress induced in laminated structures and FGMs is the main cause of structural failure [1]. The boundary element method (BEM) has distinctive advantages in solving problems of fracture mechanics [2] and elastic contact problems [3]. Until now, there have been many researchers who have used BEM to solve thermoelasticity problems with constant material properties [4-9]. But these methods are only suitable for simple temperature distribution. In order to solve thermal stress problems of a general temperature field, Gao [10] proposed a global boundary integral equation technique not needing internal cells to compute general thermoelastic problems. But the results in [10] are only suitable for solving a constant coefficient thermoelastic problem, for the important thermal structure of functionally graded materials, there is not yet presented a boundary-only BEM to deal with thermoelasticity problems with varying material properties. This paper is an effort devoted to this subject. First, boundary-domain integral equations for displacements and stresses are derived from a weighted residual form of the equilibrium equation for varying coefficient thermoelasticity. Then, the domain integrals included in the integral equations are transformed into equivalent boundary integrals using the Radial Integration Method (RIM) proposed by Gao [11] in 2002, resulting in a pure boundary element solution algorithm for varying coefficient thermal stress analysis. For numerical solving, the radial integral is very time-consuming, the radial integrals in dealing with domain integrals of varying coefficient are analytically integrated based on the use of the fourth-order spline radial basis function (RBF). Through use of the analytical expressions in RIBEM, the computational efficiency can be improved considerably. Finally, numerical examples for the thermal stress analysis of a two-dimensional (2D) system are given to verify the correctness of the derived formulations by comparison to finite element results.

\section{Displacement integral equation for varying coefficient thermoelasticity}

In thermoelasticity, the relationship between stress, displacement and temperature can be expressed as [10]

$$
\sigma_{i j}=\mu C_{i j k l}^{0} u_{k, l}-\delta_{i j} \tilde{k} \theta
$$

where

$$
\tilde{k}=\frac{2(1+v) \mu k}{1-2 v}
$$

in which, $\sigma_{i j}$ is the stress tensor, $u_{k, l}$ represents the partial derivative of displacement $u_{k}$ with respect to coordinate $x_{l}, \theta$ is the value of temperature change, $\nu$ is Poisson's ratio which is assumed to be constant in this paper, $\mu$ and $k$ are the shear modulus and thermal expansion coefficient, respectively, which are functions of spatial coordinates, and $C_{i j k l}^{0}$ is the elastic tensor with the form

$$
C_{i j k l}^{0}=\frac{2 v}{1-2 v} \delta_{i j} \delta_{k l}+\delta_{i k} \delta_{j l}+\delta_{i l} \delta_{j k}
$$


For the sake of convenience and without loss of generality, body forces are not considered in this study. Under this condition, the equilibrium of stress can be expressed as

$$
\sigma_{j k, k}=0
$$

The relationship between traction $t_{i}$ and stress is given by

$$
t_{i}=\sigma_{i j} n_{j}
$$

Using the weight function $U_{i j}$, we can write a weighted residual formulation for eqn. (4) as follows

$$
\int_{\Omega} U_{i j} \sigma_{j k, k} d \Omega=0
$$

Substituting eqn. (1) into eqn. (6), integrating by parts twice, and using Gauss's divergence theorem, it follows that

$$
\begin{aligned}
0 & =\int_{\Omega} U_{i j} \sigma_{j k, k} d \Omega=\int_{\Gamma} U_{i j} \sigma_{j k} n_{k} d \Gamma-\int_{\Gamma} U_{i j, k} C_{j k s s}^{0} n_{s} \mu u_{r} d \Gamma \\
& +\int_{\Omega}^{0} U_{i j, k s} C_{j k r s}^{0} \mu u_{r} d \Omega+\int_{\Omega} U_{i j, k} C_{j k s}^{0} \mu_{, s} u_{r} d \Omega+\int_{\Omega} U_{i j, j} \tilde{k} \theta d \Omega
\end{aligned}
$$

The weight function $U_{i j}$ is taken as the fundamental solution of the following equation

$$
U_{i j, k s} C_{j k r s}^{0}+\delta_{i r} \delta\left(\boldsymbol{x}, \boldsymbol{x}^{p}\right)=0
$$

where $\delta\left(\boldsymbol{x}, \boldsymbol{x}^{p}\right)$ is the Dirac function with the singular point at $\boldsymbol{x}^{p}$. Substituting eqn. (8) into eqn. (7) and making use of the integration property of the Dirac function, the first domain integral on the right-hand side of eqn. (7) becomes $-\mu\left(\boldsymbol{x}^{p}\right) u_{r}\left(\boldsymbol{x}^{p}\right)$. Thus, after rearranging the subscripts of eqn. (7), the following boundary-domain integral equation for displacement can be obtained.

$$
\begin{aligned}
\tilde{u}_{i}\left(\boldsymbol{x}^{p}\right) & =\int_{\Gamma} U_{i j}\left(\boldsymbol{x}, \boldsymbol{x}^{p}\right) t_{j}(\boldsymbol{x}) d \Gamma(\boldsymbol{x})-\int_{\Gamma} T_{i j}\left(\boldsymbol{x}, \boldsymbol{x}^{p}\right) \tilde{u}_{j}(\boldsymbol{x}) d \Gamma(\boldsymbol{x}) \\
& +\int_{\Omega} V_{i j}\left(\boldsymbol{x}, \boldsymbol{x}^{p}\right) \tilde{u}_{j}(\boldsymbol{x}) d \Omega(\boldsymbol{x})+\int_{\Omega} U_{i j, j}\left(\boldsymbol{x}, \boldsymbol{x}^{p}\right) \tilde{\theta}(\boldsymbol{x}) d \Omega(\boldsymbol{x})
\end{aligned}
$$

where $t_{j}$ is determined by eqn. (5) and

$$
\begin{aligned}
& T_{i j}=U_{i l, k} C_{l k j s}^{0} n_{s} \\
& V_{i j}=U_{i l, k} C_{l k j s}^{0} \tilde{\mu}_{, s}
\end{aligned}
$$

in which $\tilde{u}_{j}, \tilde{\theta}$, and $\tilde{\mu}$ are the normalized displacement, temperature, and shear modulus, respectively, defined as

$$
\begin{aligned}
& \tilde{u}_{j}=\mu u_{j} \\
& \tilde{\theta}=\tilde{k} \theta \\
& \tilde{\mu}=\ln \mu
\end{aligned}
$$

The fundamental solution $U_{i j}$ satisfying eqn. (8) turns out to be the Kelvin displacement solution with $\mu=1$. Its expression can be found in any elasticity BEM book, e.g. in [12]. $T_{i j}$ is the corresponding Kelvin traction fundamental 
solution [12] and the kernel function $V_{i j}$ can be obtained by substituting the expression of $U_{i j}$ into eqn. (10), resulting in

$$
V_{i j}=\frac{-1}{4 \pi \alpha(1-v) r^{\alpha}}\left\{\tilde{\mu}_{, k} r_{k}\left[(1-2 v) \delta_{i j}+\beta r_{, i} r_{j}\right]+(1-2 v)\left(\tilde{\mu}_{, i} r_{j}-\tilde{\mu}_{, j} r_{i}\right)\right\}
$$

The function $U_{i j, j}$ is as follows

$$
U_{i j, j}=\frac{-(1-2 v)}{4 \alpha \pi(1-v)} \frac{r_{, i}}{r^{\alpha}}
$$

in which, $\beta=2$ (2D) or $\beta=3$ (3D) and $\alpha=\beta-1$.

Eqn. (9) is the displacement integral equation formulated in terms of normalized physical quantities for thermal stress analysis. Its main feature is that the representative form is very simple and no displacement gradients are included in the integral equation. It is noted that eqn. (9) is only suitable for internal points. For boundary nodes, by taking a limit process, i.e., letting $\boldsymbol{x}^{p} \rightarrow \Gamma$, one can obtain the boundary integral equation.

\section{Stress integral equation for varying coefficient thermoelasticity}

From the first expression of eqn. (11), it follows that

$$
\frac{\partial u_{i}}{\partial x_{j}^{p}}=\frac{1}{\mu}\left(\frac{\partial \tilde{u}_{i}}{\partial x_{j}^{p}}-\tilde{u}_{i} \frac{\partial \tilde{\mu}}{\partial x_{j}^{p}}\right)
$$

Taking the partial derivative of eqn. (9) with respect to the source point $\boldsymbol{x}^{p}$, subtracting and adding a singular term [12] to regularize the strongly singular domain integral related to temperature, and then substituting the result into eqn. (14) and making use of eqn. (1), we can obtain the following stress integral equation.

$$
\begin{aligned}
\sigma_{i j}\left(\boldsymbol{x}^{p}\right)= & \int_{\Gamma} U_{i j k}\left(\boldsymbol{x}, \boldsymbol{x}^{p}\right) t_{k}(\boldsymbol{x}) d \Gamma(\boldsymbol{x})-\int_{\Gamma} T_{i j k}\left(\boldsymbol{x}, \boldsymbol{x}^{p}\right) \tilde{u}_{k}(\boldsymbol{x}) d \Gamma(\boldsymbol{x}) \\
& +\int_{\Omega} V_{i j k}\left(\boldsymbol{x}, \boldsymbol{x}^{p}\right) \tilde{u}_{k}(\boldsymbol{x}) d \Omega(\boldsymbol{x})+\int_{\Omega} \Psi_{i j}\left(\boldsymbol{x}, \boldsymbol{x}^{p}\right)\left[\tilde{\theta}(\boldsymbol{x})-\tilde{\theta}\left(\boldsymbol{x}^{p}\right)\right] d \Omega(\boldsymbol{x}) \\
& +\tilde{\theta}\left(\boldsymbol{x}^{p}\right) \int_{\Gamma} r \ln r \frac{\partial r}{\partial n} \Psi_{i j}\left(\boldsymbol{x}, \boldsymbol{x}^{p}\right) d \Gamma(\boldsymbol{x})-\delta_{i j} h \tilde{\theta}\left(\boldsymbol{x}^{p}\right)+F_{i j k}\left(\boldsymbol{x}^{p}\right) \tilde{u}_{k}\left(\boldsymbol{x}^{p}\right)
\end{aligned}
$$

in which the kernel functions $U_{i j k}$ and $T_{i j k}$ are the same as given in usual BEM books [12] by setting $\mu=1$, and $V_{i j k}$ and the free term coefficient $F_{i j k}$ are the same as described in [13]. The remaining quantities $\Psi_{i j}$ and $h$ are as follows

$$
\Psi_{i j}=\frac{(1-2 v)}{2 \alpha \pi(1-v) r^{\beta}}\left(\delta_{i j}-\beta r_{, i} r_{, j}\right)
$$




$$
h=\frac{(1+\beta)(1-2 v)}{6(1-v)}
$$

The stress integral equation (15) only holds true for internal points. For boundary nodes, hyper-singularity may occur when the source point $\boldsymbol{x}^{p}$ approaches the field point $\boldsymbol{x}$.

\section{Converting domain integrals to the boundary using RIM}

To avoid discretizing the computational domain into cells in order to evaluate the domain integrals appearing in eqns. (9) and (15), the radial integration method (RIM) $[10,11]$ is applied to convert these domain integrals into equivalent boundary integrals and results in a boundary-only element algorithm. However, in general thermal stress analysis, the temperature distribution over the computation region is obtained through solving heat conduction differential equations under certain thermal boundary conditions. The obtained results are temperature values at discretized nodes. The function relationship of temperature is usually obtained by a function approximation scheme. The augmented radial basis function approximation is proved to be a good option [11]. Thus, the normalized temperature $\tilde{\theta}$ appearing in the domain integrals of eqn. (9) and eqn. (15) is approximated as

$$
\begin{gathered}
\tilde{\theta}(\boldsymbol{x})=\sum_{A} \alpha^{A} \varphi^{A}\left(R / S_{A}\right)+a^{k} x_{k}+a^{0} \\
\sum_{A} \alpha^{A}=0, \quad \sum_{A} \alpha^{A} x_{i}^{A}=0
\end{gathered}
$$

in which, $R=\left\|\boldsymbol{x}-\boldsymbol{x}^{A}\right\|$ is the distance from the application point $\boldsymbol{x}^{A}$ to the field point $x, \alpha^{A}$ and $a^{k}$ are coefficients to be determined by nodal temperature values through a node allocation scheme, and $\varphi^{A}$ is the radial basis function. Numerical investigation indicates that the 4 th order spline-type radial basis function can give very stable results [13], its expression being as follows

$$
\varphi^{A}\left(R / S_{A}\right)= \begin{cases}1-6\left(\frac{R}{S_{A}}\right)^{2}+8\left(\frac{R}{S_{A}}\right)^{3}-3\left(\frac{R}{S_{A}}\right)^{4} & 0 \leq R \leq S_{A} \\ 0 & R \geq S_{A}\end{cases}
$$

where $S_{A}$ is the support size for the application point A. Substituting eqn. (18) into the domain integral of eqn. (9) and applying RIM formulations [10, 11] yield

$$
\int_{\Omega} U_{i j, j} \tilde{\theta} d \Omega=\sum_{A} \alpha^{A} \int_{\Gamma} U_{i j, j} \frac{\partial r}{\partial n} F^{A} d \Gamma+\frac{a^{k}}{2} \int_{\Gamma} U_{i j, j} r_{, k} r^{2} \frac{\partial r}{\partial n} d \Gamma+\left(a^{k} x_{k}^{p}+a^{0}\right) \int_{\Gamma} U_{i j, j} r \frac{\partial r}{\partial n} d \Gamma
$$

To evaluate the radial integrals shown in eqns. (9) and (15), the following relationships are used: 


$$
R=\sqrt{r^{2}+2 s r+\bar{R}^{2}} \quad \bar{R}_{i}=x_{i}^{p}-x_{i}^{A}
$$

where $x_{k}^{p}$ is the coordinate at the source point, and

$$
F^{A}=\int_{0}^{r} \varphi^{A} d r
$$

$F^{A}$ can be analytically integrated as described in [14].

Similarly, using RIM, the domain integral including temperature in eqn. (15) can be transformed into the following boundary integral.

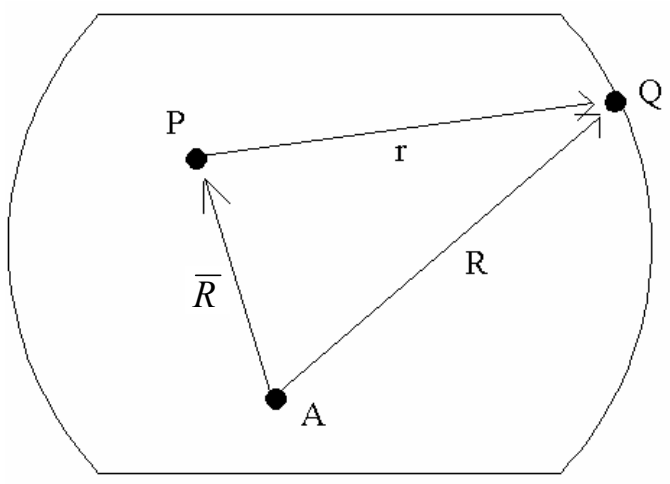

Figure 1: Relationship between distances.

$$
\begin{gathered}
\int_{\Omega} \Psi_{i j}\left[\tilde{\theta}-\tilde{\theta}\left(\boldsymbol{x}^{p}\right)\right] d \Omega=\sum_{A} \alpha^{A} \int_{\Gamma} \Psi_{i j} r \frac{\partial r}{\partial n} F^{A} d \Gamma+a^{k} \int_{\Gamma} \Psi_{i j} r_{k} r^{2} \frac{\partial r}{\partial n} d \Gamma \\
F^{\prime A}=\int_{0}^{r} \frac{\varphi^{A}-\varphi^{A}\left(\boldsymbol{x}^{p}\right)}{r} d r
\end{gathered}
$$

Referring to fig. $1, F^{\prime A}$ can be analytically integrated as:

$$
\begin{aligned}
F^{A}=\int_{0}^{r} & \frac{\varphi^{A}-\varphi^{A}\left(\boldsymbol{x}^{p}\right)}{r} d r=\int_{r_{1}}^{r_{2}} \frac{1-6\left(\frac{R}{S_{A}}\right)^{2}+8\left(\frac{R}{S_{A}}\right)^{3}-3\left(\frac{R}{S_{A}}\right)^{4}-\varphi^{A}\left(\boldsymbol{x}^{p}\right)}{\mathrm{r}} \mathrm{d} r \\
= & \left\{\left[(-1) \times\left(0.75 r^{4}+12\left(S_{A}^{2}+\bar{R}^{2}\right) r s+3 r^{2}\left(S_{A}^{2}+\bar{R}^{2}+2 s^{2}\right)\right.\right.\right. \\
& -\frac{4}{3} S_{A}\left(8 \bar{R}^{2}+2 r^{2}+7 r s+3 s^{2}\right) \sqrt{r^{2}+2 s r+\bar{R}^{2}}+4 r^{3} s \\
& +4 s S_{A}\left(s^{2}-3 \bar{R}^{2}\right) \log \left(r+s+\sqrt{r^{2}+2 s r+\bar{R}^{2}}\right) \\
& \left.\left.+8 S_{A} \bar{R}^{3} \log \left(\bar{R}^{2}+r s+\bar{R} \sqrt{r^{2}+2 s r+\bar{R}^{2}}\right)\right] / S_{A}^{4}\right)\left.\right|_{r 2} ^{r 1}
\end{aligned}
$$


where $r_{1}$ and $r_{2}$ are the intersections between the line $r$ and the circle centered at $A$ with radius $S_{A}$ of the supported region, as shown in fig. 2.

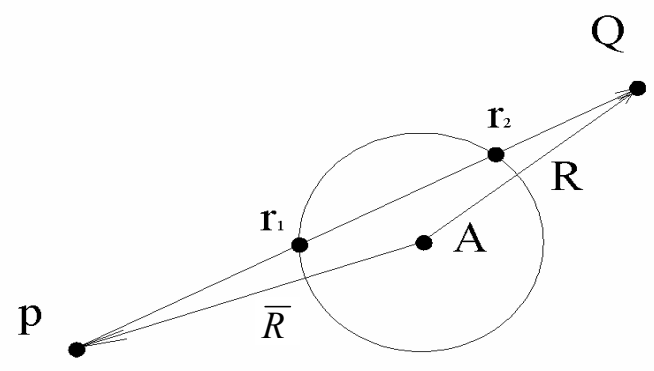

Figure 2: Intersections between line and circle.

For the expression as shown in eqn. (26), it should be noted that special case, $r+s+\sqrt{r^{2}+2 s r+\bar{R}^{2}}=0$ and $\bar{R}^{2}+r s+\bar{R} \sqrt{r^{2}+2 s r+\bar{R}^{2}}=0$ may happen. In these cases, $s=-\bar{R}$, as shown in fig. 3, $R$ degenerates to $R=\sqrt{r^{2}+2 s r+\bar{R}^{2}}=|\bar{R}-r|$ and $F^{\prime A}$ can be integrated as

$$
\begin{aligned}
F^{\prime A} & =\int_{0}^{r} \frac{\varphi^{A}-\varphi^{A}\left(x^{p}\right)}{r} d r \int_{0}^{r}=\left(-0.75 r^{4}+12\left(S_{A}^{2}+\bar{R}^{2}\right) \bar{R} r+4 r^{3} \bar{R}-3 r^{2}\left(S_{A}^{2}+3 \bar{R}^{2}\right)\right. \\
& +\frac{4}{3} S_{A}|\bar{R}-r| r\left(18 \bar{R}^{2}+2 r^{2}-9 \bar{R} r\right) /(r-\bar{R})+8 S_{A} \bar{R}^{3}|\bar{R}-r| \log (r) /(\bar{R}-r) \\
& \left.-8 S_{A} \bar{R}^{3} \log (r)\right) / S_{A}^{4}
\end{aligned}
$$

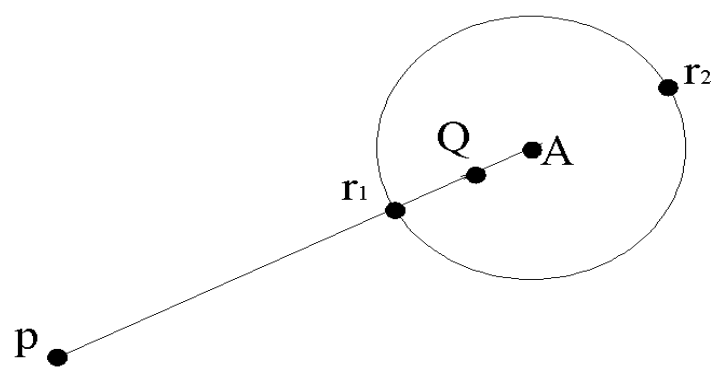

Figure 3: A special intersection.

Then, substituting eqns. (27) and (23) into the above equation and the results into eqns. (9) and (15) for boundary and internal points, one can establish the desired system of equations. 


\section{Numerical examples}

The displacement and stress integral equations (9) and (15) derived in this paper have been built in the three-step multi-domain BEM solver developed by Gao et al. [15] for solving non-homogeneous problems. Also, based on the program BEMECH listed in reference [12], a powerful computer code named RIBEM have been developed for performing thermal stress analysis consisting of an arbitrary number of FGMs. The numerical examples for $2 \mathrm{D}$ thermal stress analysis are provided in the following section to demonstrate the correctness of the derived formulation.

\subsection{Thermal stress analysis of a rectangular plate}

This example is concerned with a $200 \times 100$ rectangular plate. The BEM model consists of a total of 60 linear boundary elements and 231 nodes, among which there are 60 boundary nodes, and 171 internal nodes as shown in fig. 4. To obtain the temperature distribution over the rectangular plate, the RIBEM is first used to perform the heat conduction computation and then the thermal stress analysis is carried out using the computed temperature. The boundary conditions (BC) for heat conduction computation are as shown in fig. 5: the adiabatic condition $(\mathrm{q}=0)$ is imposed on the top and bottom sides, temperatures of $0 \mathrm{~K}$ and $100 \mathrm{~K}$ are applied to the left and right sides, respectively. The varying heat conductivity along the y-direction of $k=k_{1} e^{\gamma y}$ where $\gamma=\frac{1}{H} \ln \frac{k_{2}}{k_{1}}$ in which $\mathrm{H}=100$ is the width of the plate, and $k_{1}=100$ and $k_{2}=200$ are the conductivities on the bottom and top sides of the plate, respectively. The $\mathrm{BC}$ for thermal stress analysis is as follows: the left side is fixed and other sides are traction free.

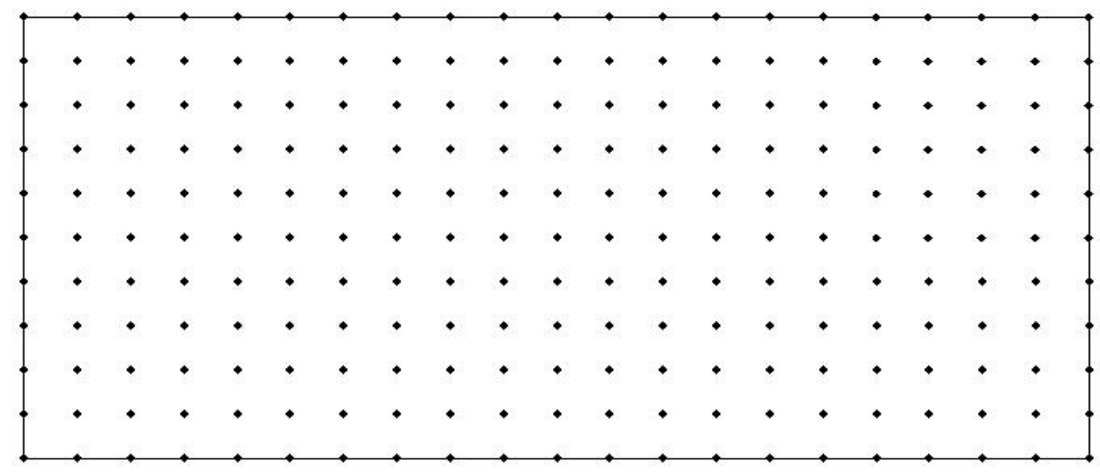

Figure 4: $\quad$ BEM mesh of the plate. 


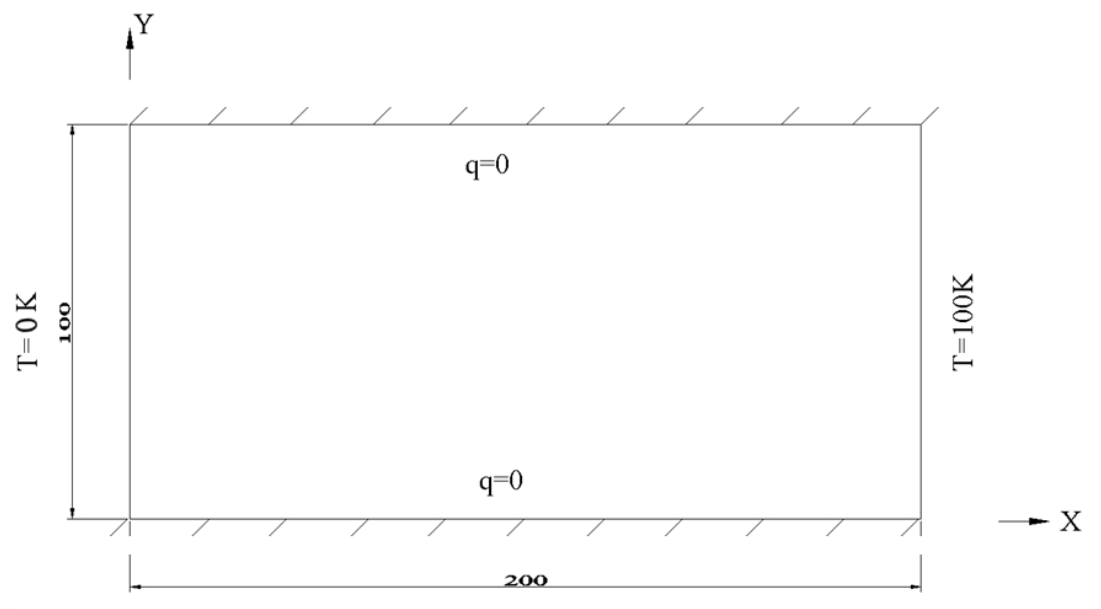

Figure 5: Dimension of the plate.

For comparison, this problem is also computed using the FEM software ANSYS. Figure 6 shows the distribution of the displacement $U_{x}$ along the $y=50$ central line, obtained using RIBEM and FEM. fig. 7 is the computed stress $\sigma_{11}$ along the middle line $x=100$ of the plate, while figs. 8 and 9 plot the temperature and displacement contours after deformation. From figs. 6 and 7, it can be seen that the BEM results are very close to those of FEM. This indicates that the formulations derived in this paper are correct. In this example, the computational time using numerical integration is 4 seconds, while analytical integration RIBEM takes 2 seconds. About $50 \%$ of computational time is saved.

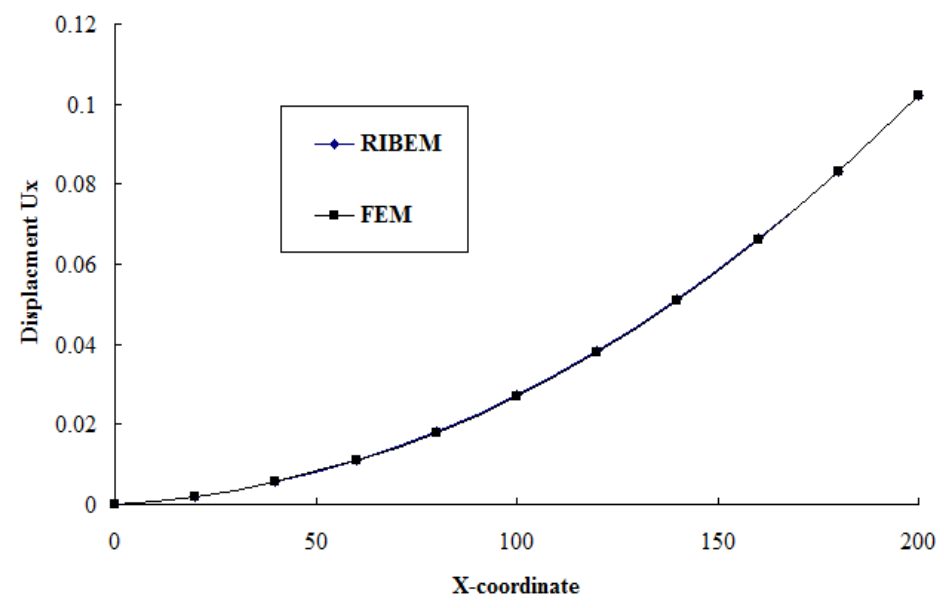

Figure 6: Displacement distribution along central line of the plate. 


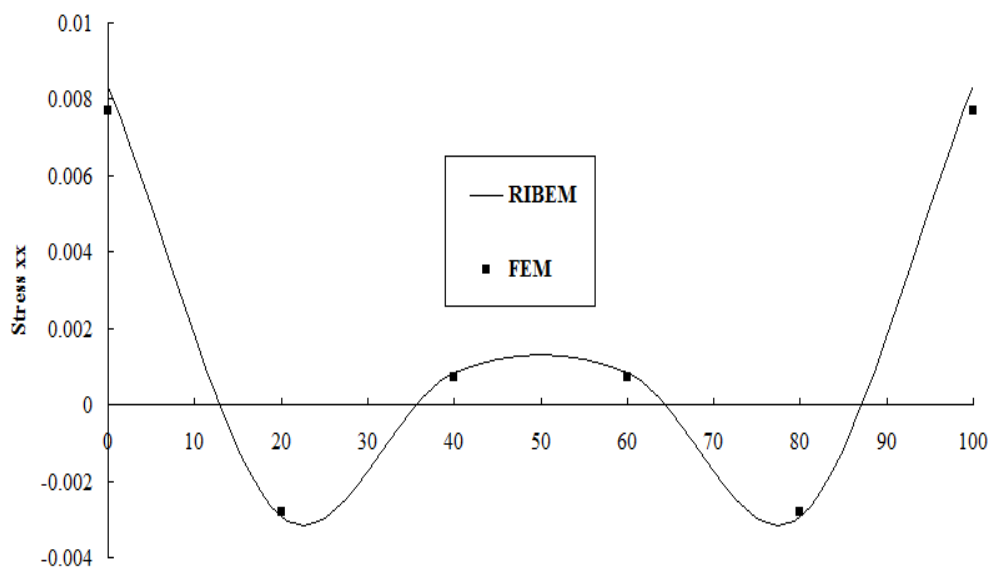

Y-coordinate

Figure 7: $\quad$ Stress distribution along $x=100$ of the plate.

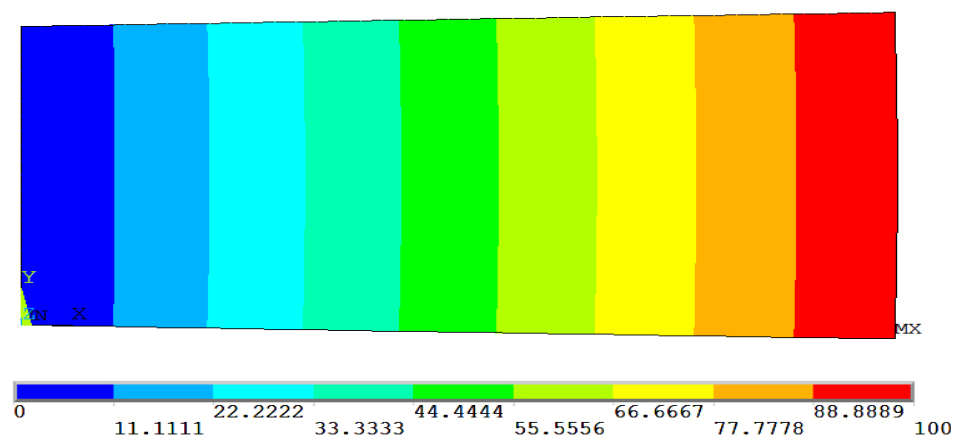

Figure 8: $\quad x$ direction temperature contour after deformation.
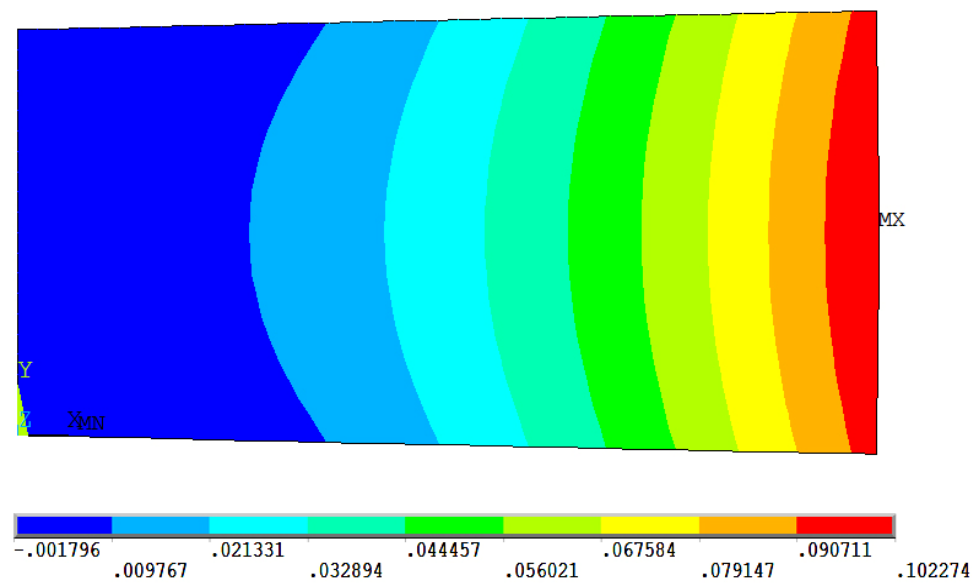

Figure 9: $\quad x$ direction displacement contour after deformation. 


\section{Conclusions}

A new set of displacement and stress boundary-domain integrals are derived for thermal stress analysis of functionally graded materials. These equations have been coded into a three-step multi-domain BEM solver, which is able to perform thermoelasticity analysis for complicated structures consisting of arbitrary numbers of materials. Attributed to the use of normalized displacement (the product of shear modulus and displacement) and normalized temperature (the product of the thermal expansion coefficient and temperature) to formulate the integral equations, the derived formulations are very simple in form and do not contain any displacement gradients. The domain integrals involved in the integral equations due to the non-homogeneity of FGMs have been converted to the boundary using RIM and result in a boundary-only element algorithm. For numerical solving the radial integral is very time-consuming, the radial integrals in dealing with domain integrals of varying coefficient are analytically integrated based on the use of the fourth-order spline radial basis function (RBF). Through use of the analytical expressions in RIBEM, the computational efficiency can be improved considerably. Numerical example has been analyzed using both the presented BEM and FEM. The comparison of computational results from the two methods has verified the correctness of the derived formulations.

\section{Acknowledgement}

The authors gratefully acknowledge the National Natural Science Foundation of China for financial support to this work under Grant NSFC No. 11172055, 11202045 .

\section{References}

[1] Suresh, S and Mortensen, A. Fundamentals of Functionally Graded Materials. IOM Communications Ltd., London, 1998.

[2] Rudas, M. and Bush, M.B., Evolution of subsurface radial cracks in bimaterial structures undergoing indentation loading. Engineering Analysis with Boundary Elements, 31, pp. 66-74, 2007.

[3] Yao, Z.H. and Liu, Y.J., A direct error estimation of BEM for 3D elastic contact problem. Journal of Yanshan University, 28(2), pp. 95-98, 2004.

[4] Rizzo, F.J. and Shippy, D.J., An advanced boundary integral equation method for three-dimensional thermoelasticity. Int. J. Numer. Meth. Engng, 11, pp. 1753-1768, 1977.

[5] Henry, Jr.D.P. and Banerjee, P.K., A new boundary element formulation for two- and three-dimensional thermoelasticity using particular integrals. International Journal for Numerical Methods in Engineering, 26, pp. 2061-77, 1988.

[6] Karami, G. and Kuhn, G., Implementation of thermoelastic forces in boundary element analysis of elastic contact and fracture mechanics 
problems. Engineering Analysis with Boundary Elements, 10, pp. 13-22, 1992.

[7] Shiah, Y.C. and Tan, C.L., Exact boundary integral transformation of the thermoelastic domain integral in BEM for general 2D anisotropic elasticity. Comput. Mech, 23, pp. 87-96, 1999.

[8] Cheng, A.H.D., Chen, C.S., Golberg, M.A. and Rashed, Y.F., BEM for thermoelasticity and elasticity with body force - a revisit. Engineering Analysis with Boundary Elements, 25, pp. 377-387, 2001.

[9] Gao, X.W., Zhang, Ch., Sladek, J. and Sladek, V., Fracture analysis of functionally graded materials by a BEM. Composites Science and Technology, 68, pp. 1209-1215, 2008.

[10] Gao, X.W., Boundary element analysis in thermoelasticity with and without internal cells. Int. J. Numer. Meth. Engng, 57, pp. 975-990, 2003.

[11] Gao, X.W., A boundary element method without internal cells for twodimensional and three-dimensional elastoplastic problems. ASME Journal of Applied Mechanics, 69, pp. 154-160, 2002.

[12] Gao, X.W. and Davies TG., Boundary Element Programming in Mechanics. Cambridge University Press 2002, (ISBN: 052177359-8).

[13] Gao, X.W., Zhang, Ch. and Guo, L., Boundary-only element solutions of 2D and 3D nonlinear and nonhomogeneous elastic problems. Engineering Analysis with Boundary Elements, 31, pp. 974-982, 2007.

[14] Yang K, Gao XW and Liu YF. Using analytical expressions in radial integration BEM for variable coefficient heat conduction problems. Engineering Analysis with Boundary Elements, 35, pp. 1085-1089, 2011.

[15] Gao, X.W., Guo, L. and Zhang, Ch., Three-step multi-domain BEM solver for nonhomogeneous material problems. Engineering Analysis with Boundary Elements, 31, pp. 965-973, 2007. 